\title{
CLINICAL RESEARCH ARTICLE OPEN Genetic and metabolic predictors of hepatic fat content in a cohort of Italian children with obesity
}

Alessia Di Costanzo (iD) , Lucia Pacifico ${ }^{2}$, Claudio Chiesa ${ }^{3}$, Francesco Massimo Perla ${ }^{2}$, Fabrizio Ceci ${ }^{4}$, Antonio Angeloni ${ }^{4}$, Laura D’Erasmo ${ }^{1}$, Michele Di Martino ${ }^{5}$ and Marcello Arca ${ }^{1}$

OBJECTIVES: To comprehensively explore metabolic and genetic contributors to liver fat accumulation in overweight/obese children.

METHODS: Two hundred thirty Italian children with obesity were investigated for metabolic parameters and genotyped for PNPLA3, TM6SF2, GCKR, and MBOAT7 gene variants. Percentage hepatic fat content (HFF\%) was measured by nuclear magnetic resonance.

RESULTS: HFF\% was positively related with BMI, HOMA ${ }_{I R}$, metabolic syndrome, ALT, AST, $\gamma$ GT, and albumin. Carriers of [G] allele in PNPLA3, [T] allele in GCKR and [T] allele in TM6SF2 genes had significantly higher hepatic fat content than wild-type carriers. HFF\% was explained for $8.7 \%$ by metabolic and for $16.1 \%$ by genetic factors and, a model including age, gender, BMI, HOMA $\mathrm{IR}_{2}, P N P L A 3$, GCKR, and TM6SF2 variants was the best predictor of HFF\%, explaining $24.8 \%$ of its variation $(P<0.001)$. A weighted-genetic risk score combining PNPLA3, GCKR, and TM6SF2 risk alleles was associated with almost eightfold higher risk of NAFLD.

CONCLUSIONS: Our data highlighted the predominant role of genetic factors in determining the amount of liver fat content in children with obesity.

Pediatric Research (2019) 85:671-677; https://doi.org/10.1038/s41390-019-0303-1

\section{INTRODUCTION}

Paralleling the worldwide epidemic of obesity in childhood, nonalcoholic fatty liver disease (NAFLD) has become the most common liver abnormality in children and adolescents. ${ }^{1}$ Indeed, it has been reported that NAFLD can be detected in up to $60 \%$ of overweight children. ${ }^{2-5}$ However, the fact that not all children with obesity develop NAFLD suggests that environmental and/or genetic factors may confer NAFLD susceptibility to each obese individual.

NAFLD predisposes to a broad spectrum of chronic liver diseases mostly occurring in adulthood, such as non-alcoholic steatohepatitis (NASH), cirrhosis, and hepatocellular carcinoma. ${ }^{6,7}$ Therefore, the identification of factors promoting increased liver fat content in children with obesity is clinically relevant as it may favor early preventive interventions in high-risk individuals. In addition, the recognition of these relationships at early stage may allow the better understanding of the steatogenic role of different factors since there is less potential for confounders (e.g., drug, alcohol consumption).

Several reports have recognized the leading role of insulin resistance in promoting hepatic fat content (HFF) in obesity, even though it has not been definitively established whether this condition may represent the cause or the consequence of the increased HFF in the liver. ${ }^{8,9}$ In addition, several susceptibility gene variants have been identified. ${ }^{10,11}$ In particular, the rs738409 C>G sequence variant in the Patatin-like Phospholipase domaincontaining 3 (PNPLA3) gene, encoding for the 1148M protein variation, has been identified as a major determinant of the interindividual and ethnicity-related differences in HFF in adults. ${ }^{12,13}$ The mechanisms by which this substitution induces liver fat is related to an impaired hepatocellular triglycerides hydrolysis and increased lipogenesis associated to the $148 \mathrm{M}$ allele. ${ }^{14}$ The Transmembrane 6 Superfamily Member 2 (TM6SF2) gene has also been shown to increase NAFLD susceptibility causing an impaired mobilization of neutral lipids for very low-density lipoprotein assembly and secretion by the liver in rs58542926 C>T (E167K) carriers. ${ }^{15,16}$ Other pro-steatogenic variants have been identified in the Protein Phosphatase 1 Regulatory Subunit 3B (PPP1R3B), the Glucokinase Regulator (GCKR), Neurocan (NCAN), Lysophospholipase-Like 1 (LYPLAL1), and the Membrane Bound O-acyltransferase domaincontaining 7 (MBOAT7-TMC4) genes, but their involvement in the pathogenesis of liver steatosis is less firmly established. ${ }^{17,18}$ We have recently reported that a genetic score based on PNPLA3, TM6SF2, $G C K R$, and MBOAT7 variants was highly predictive of NAFLD in adults. ${ }^{19}$ However, only some of these susceptibility genes have received direct confirmation in children. ${ }^{20-23}$

Thus, in this study we present a comprehensive evaluation of genetic and metabolic factors influencing HFF in a cohort of Italian children with obesity. As the sensitivity of ultrasound techniques is limited, we have used magnetic resonance imaging (MRI) to obtain an accurate quantitative assessment of the amount of liver fat.

\section{METHODS}

Study subjects

This observational study included 230 overweight [body mass index $(\mathrm{BMI})>85$ th and $<95$ th percentile for age and gender] or

\footnotetext{
${ }^{1}$ Department of Internal Medicine and Medical Specialties, Sapienza University of Rome, 00161 Rome, Italy; ${ }^{2}$ Department of Pediatrics, Sapienza University of Rome, 00161 Rome, Italy; ${ }^{3}$ Institute of Translational Pharmacology, National Research Council, 00133 Rome, Italy; ${ }^{4}$ Department of Experimental Medicine, Sapienza University of Rome, 00161 Rome, Italy and ${ }^{5}$ Department of Radiology, Sapienza University of Rome, 00161 Rome, Italy

Correspondence: Alessia Di Costanzo (alessia.dicostanzo@uniroma1.it)
} 
with obesity (BMI $\geq 95$ th percentile for age and gender) children and adolescents (aged 6-16 years), who were consecutively admitted at the outpatient services of the Department of Pediatrics, Sapienza University of Rome, ${ }^{24,25}$ to receive a clinical evaluation for obesity. All children were Caucasians and ethnically homogeneous.

All study children had a complete physical examination as reported in detail elsewhere. ${ }^{25}$ The pubertal status was assessed according to Tanner. ${ }^{26}$ The degree of obesity was quantified using Cole's least mean-square method, which normalizes the skewed distribution of BMI and expresses BMI as standard deviation (SD) score. ${ }^{24,25}$ Children with history of diabetes and endocrine or renal disease were excluded. Those with secondary causes of steatosis, including hepatic virus infections (hepatitis A-E and G, cytomegalovirus, and Epstein-Barr virus), autoimmune hepatitis, metabolic liver disease, a-1-antitrypsin deficiency, cystic fibrosis, Wilson's disease, hemochromatosis, and celiac disease were also excluded. ${ }^{25}$ Metabolic syndrome (MetS) was diagnosed in the presence of any three of the following: (1) WC $\geq 90$ th age- and sexspecific percentiles according to reference curves for children aged $2-18$ years; ${ }^{27,28}$ (2) elevated systolic and diastolic BP $\geq 90$ th percentile for age, gender, and height; ${ }^{29}$ (3) low high-density lipoprotein cholesterol (HDL) values ( $\leq 10$ th) according to reference curve for all ages/sexes; ${ }^{27,28}$ (4) elevated triglycerides (TG) values $\geq 90$ th sex and age-specific percentile according to reference curves for children aged $2-18$ years, ${ }^{27,28}$ and (5) fasting glucose $\geq 5.6 \mathrm{mmol} / \mathrm{L}^{27}$

The study protocol was reviewed and approved by the Ethics Committee of Policlinico Umberto I Hospital (EC approval \#2464). Written informed consent was obtained from the next of kin, caretakers, or guardians on behalf of the children enrolled in this study, in accordance with principles of Helsinki Declaration.

Liver magnetic resonance imaging (MRI)

MRI data were acquired on a $3.0 \mathrm{~T}$ MR scanner with a $50 \mathrm{mT} / \mathrm{m}$ maximum gradient length and $200 \mathrm{~T} / \mathrm{m} / \mathrm{s}$ maximum slew rate (Discovery MR 750; GE Medical Systems, Milwaukee, WI) using an eight-element body torso-array coil system. ${ }^{30-32}$ All spectra were obtained in the stimulated echo acquisition mode, using a breath hold sequence with an acquisition time of $\sim 24 \mathrm{~s}^{32}$ HFF\% was measured as previously described and validated. ${ }^{30-32}$ According to results of previous investigations, children showing HFF $\geq 5 \%$ were classified as having NAFLD. ${ }^{31,32}$

\section{Laboratory measurements}

Blood samples were taken from all study subjects after an overnight fast for estimation of glucose, insulin, total cholesterol (TC), HDL-C, TG, alanine aminotransferase (ALT), aspartate aminotransferase (AST), and gamma-glutamyl transferase $(\gamma \mathrm{GT}){ }^{25}$ An oral glucose tolerance test (OGTT) was performed for all overweight/obese children as yet reported. ${ }^{30}$ Insulin sensitivity were estimated through the homeostasis model assessment $\left(\mathrm{HOMA} A_{\mathrm{IR}}\right){ }^{30}$ We also classified subjects as having elevated ALT using cut off values established from NHANES data in boys ( $>25.8$ $\mathrm{U} / \mathrm{L}$ ) and girls (>22.1 U/L). ${ }^{33}$ All analyses were conducted by COBAS 6000 (Roche Diagnostics). ${ }^{30}$ Insulin concentrations were measured by an electrochemiluminescent method. ${ }^{25}$

\section{DNA genotyping}

Genomic DNA was extracted from whole blood according standard procedures. The rs641738 C>G (I148M) (PNPLA3), rs58542926 C>T (E167K) (TM6SF2), rs1260326 C>T (L446P) (GCKR), and rs641738 C>T (G17E) (MBOAT7-TMC4) were considered for genotyping. They were selected because in a previous resequencing study carried out in an Italian adult cohort they emerged as significant genetic determinants of NAFLD. ${ }^{19}$ Genotyping were performed in duplicate by TaqMan 5'-Nucleotidase assay. ${ }^{19,34}$
Genetic risk score computation

The Genetic Risk Score (GRS) was calculated based on the four selected SNPs. As previously described, two methods were used: (1) a simple counting method (unweighted GRS) and (2) a weighted method (weighted GRS). ${ }^{19}$ The $\beta$-coefficients considered for weighted GRS calculation and each SNP were 0.2653 (rs738409), 0.2711 (rs58542926), 0.0649 (rs1260326), and 0.0575 (rs641738). ${ }^{35}$ The GRS was modeled as a continuous variable and then categorized into tertiles. ${ }^{19}$

\section{Statistical analysis}

Statistical analyses were performed by using the SPSS package (version 22.0), SPSS Inc., Chicago, IL. Data were reported as means and standard deviations for normally distributed variables, or as median and interquartile range for non-normally distributed variables. Differences between groups were evaluated by $t$-test or Mann-Whitney $U$-test, as appropriate. Proportions were compared by the $X^{2}$ test.

Genotype frequencies were assessed for Hardy-Weinberg equilibrium (HWE) using the goodness-of-fit $x^{2}$ test. It must be noted that because of the low frequency of the T allele (167K) in TM6SF2 gene (only one homozygous subject), all calculations were based on the dominant model of inheritance.

Predictors of HFF\% were evaluated by linear regression analysis by adding once at time each independent variable. $R^{2}$-value and $\beta$-coefficients were used to evaluate the percentage of variation of HFF explained by each variable and the degree of change in HFF\% for every 1-unit of change of the independent variable. The association of PNPLA3, GCKR, TM6SF2, and MBOAT7 genotypes with HFF\% was evaluated by a linear regression analysis adjusted for age, gender, BMI, pubertal stage, and $\mathrm{HOMA}_{\mathrm{IR}}$. Finally, genetic and non-genetic variables, which emerged as significantly associated to HFF\%, were then included into multivariate linear regression models to determine their independency in predicting HFF\%. Age, gender, BMI, and $\mathrm{HOMA}_{\mathrm{IR}}$ were entered in the first model followed by PNPLA3, $G C K R$, and TM6SF2 variants (Enter method). $R^{2}$-value, variation of $R^{2}, F$-test, and $P$-value $F$-test were used to identify the best model able to predict HFF\% variation and the significant amount of variance in HFF\%. Collinear variables, evaluated by Pearson bivariate analysis, were excluded. Logistic regression analysis was used to examine the odds ratios (ORs) of NAFLD associated to metabolic and genetic variables and weighted GRS. ORs were adjusted for age, gender, BMI, and $\mathrm{HOMA}_{\mathrm{IR}}$. Multiple comparisons were further adjusted by bootstrap correction based on 1000 bootstrap samples. Statistical significance was taken at a nominal $P$-value $<0.05$ for all comparisons.

\section{RESULTS}

Clinical characteristics of study population

Baseline characteristics are shown in Table 1. One hundred and thirty-one were boys. The mean age was $10.2 \pm 3.0$ years. Overall, $86 \%$ showed obesity as defined by sex- and genderspecific $B M I$ values $\geq 95$ th percentile. Measures of adiposity, most notably BMI $(P=0.010)$ and WC $(P=0.004)$, were found to be higher in boys than girls. In the entire cohort $47.1 \%$ of children were classifiable as insulin resistant according to a previously reported criterion $\left(\mathrm{HOMA}_{\mathrm{IR}} \geq 2.5\right) .{ }^{36}$ Systolic and diastolic BP as well as plasma lipids were within normal ranges and did not differ between genders, even after adjustment for age, BMI, and pubertal stage. Overall, $19.8 \%$ of boys and $14.1 \%$ of girls showed the characteristics of MetS $(P=0.25$ for gender difference).

The distribution of HFF\% values in the entire study cohort is shown in the Supplemental Figure S1 (online). Median HFF\% was $3.0 \%$ (interquartile range $1-10 \%$ ) and $45.7 \%$ of children showed 
Table 1. Clinical characteristics of the study population

\begin{tabular}{|c|c|c|c|c|}
\hline & All & Boys & Girls & $P$ \\
\hline$N$ & 230 & 131 & 99 & \\
\hline Age (years) & $10.2 \pm 3.0$ & $10.7 \pm 3.1$ & $9.5 \pm 2.7$ & 0.003 \\
\hline \multicolumn{5}{|l|}{ Body composition } \\
\hline $\mathrm{BMI}\left(\mathrm{kg} / \mathrm{m}^{2}\right)$ & $25.4(23.1-28.7)$ & $26.0(23.4-29.5)$ & $24.6(22.8-27.1)$ & 0.010 \\
\hline BMI Z-score & $2.04(1.8-2.3)$ & $2.1(1.8-2.3)$ & $2.0(1.8-2.2)$ & 0.36 \\
\hline$W C(\mathrm{~cm})$ & $86.9 \pm 11.6$ & $88.8 \pm 11.6$ & $84.4 \pm 11.1$ & 0.004 \\
\hline $\mathrm{BMI} \geq 95$ th $(n, \%)$ & $196(86)$ & $87(83.7)$ & 109 (87.9) & 0.88 \\
\hline Pubertal stage & & & & 0.76 \\
\hline Stage 1 & $88(38.3)$ & $52(39.7)$ & $36(36.4)$ & 0.09 \\
\hline Stage 2 & $59(25.7)$ & $30(22.9)$ & $29(29.3)$ & 0.89 \\
\hline Stage 3 & $33(14.3)$ & $21(16.0)$ & $12(12.1)$ & 0.12 \\
\hline Stage 4 & $21(9.1)$ & $12(9.2)$ & $9(9.1)$ & 0.51 \\
\hline Stage 5 & $28(12.2)$ & $15(11.5)$ & $13(13.1)$ & 0.70 \\
\hline MetS $(n, \%)^{\mathrm{a}}$ & $40(17.4)$ & $26(19.8)$ & $14(14.1)$ & 0.26 \\
\hline HFF (\%) & $3.0(1-10)$ & $5.0(1-10)$ & $2.0(1-7)$ & 0.042 \\
\hline $\operatorname{NAFLD}(n, \%)^{\mathrm{b}}$ & $105(45.7)$ & $68(51.9)$ & 37 (37.4) & 0.028 \\
\hline \multicolumn{5}{|l|}{ Blood pressure } \\
\hline Systolic (mmHg) & $111.5(106-120)$ & $113.3(106-120)$ & $111(105-120)$ & 0.80 \\
\hline Diastolic (mmHg) & $65(60-70)$ & $65.0(60.0-70.0)$ & $65.0(60-70)$ & 0.95 \\
\hline \multicolumn{5}{|l|}{ Plasma lipids } \\
\hline $\mathrm{TC}(\mathrm{mg} / \mathrm{dL})$ & $160.4 \pm 31.9$ & $161.4 \pm 34.0$ & $159.2 \pm 29.04$ & 0.60 \\
\hline LDL-C (mg/dL) & $92.9 \pm 27.6$ & $93.2 \pm 29.1$ & $92.5 \pm 25.6$ & 0.80 \\
\hline $\mathrm{TG}(\mathrm{mg} / \mathrm{dL})$ & $75.0(51-106)$ & $72.5(51-106)$ & $75.0(51.5-107)$ & 0.86 \\
\hline $\mathrm{HDL}-\mathrm{C}(\mathrm{mg} / \mathrm{dL})$ & $50 \pm 12.6$ & $50.7 \pm 13.4$ & $52.1 \pm 13.5$ & 0.44 \\
\hline APOB $(\mathrm{mg} / \mathrm{dL})$ & $0.46(0.64-0.88)$ & $0.74(0.63-0.85)$ & $0.79(0.65-0.89)$ & 0.17 \\
\hline APOAl (mg/dL) & $1.38(1.25-1.54)$ & $1.39(1.26-1.54)$ & $1.36(1.23-1.54)$ & 0.33 \\
\hline \multicolumn{5}{|c|}{ Measures of glucose homeostasis } \\
\hline \multicolumn{5}{|c|}{ Blood glucose (mg/dL) } \\
\hline Fasting & $83(77-86)$ & $83.0(79.0-86.0)$ & $81.0(77.0-86.0)$ & 0.06 \\
\hline $120 \min$ & $95.2 \pm 16.7$ & $97.0 \pm 17.7$ & $92.5 \pm 14.8$ & 0.06 \\
\hline \multicolumn{5}{|l|}{ Insulin (UI/L) } \\
\hline Fasting & $11.8(8-16.9)$ & $11.8(7.8-17.1)$ & $11.8(9.2-16.7)$ & 0.61 \\
\hline $120 \mathrm{~min}$ & $42.4(23.3-68.8)$ & $41.3(26.6-72.6)$ & $42.7(21.7-62.9)$ & 0.53 \\
\hline $\mathrm{HOMA}_{\mathrm{IR}}$ & $2.4(1.6-3.6)$ & $2.4(1.5-3.6)$ & $2.3(1.8-3.6)$ & 0.62 \\
\hline \multicolumn{5}{|l|}{ Liver function tests } \\
\hline ALT (UI/L) & $20.5(16-28.2)$ & $22(17-31)$ & $19(15-24)$ & 0.005 \\
\hline AST (UI/L) & $23(20-27)$ & $24(21-27)$ & $23(20-26.5)$ & 0.28 \\
\hline AST/ALT ratio & $1.10(0.83-1.41)$ & $1.05(0.76-1.35)$ & $1.17(0.92-1.51)$ & 0.006 \\
\hline GGT (UI/L) & $14(11-18)$ & $14(12-19)$ & $12(10-16)$ & 0.002 \\
\hline Albumin (g/L) & $49.0(47-50.5)$ & $49.0(47-51)$ & $48(46-50)$ & 0.02 \\
\hline Ferritin $(\mu \mathrm{g} / \mathrm{L})$ & $66.2 \pm 35.8$ & $68.7 \pm 39.01$ & $62.8 \pm 30.9$ & 0.35 \\
\hline CRP $(\mu \mathrm{g} / \mathrm{L})$ & $1600(800-3600)$ & $1400(700-2850)$ & $1800(900-4600)$ & 0.010 \\
\hline
\end{tabular}

Data are expressed as percentage, mean $( \pm S D)$ and median (25th-75th percentile range) as appropriate

$A P O A 1$ Apoliporotein $\mathrm{A}-\mathrm{I}, A P O B$ apoliporotein $\mathrm{B}, C R P$ C-reactive protein, $L D L$ -

$C$ low-density lipoprotein cholesterol

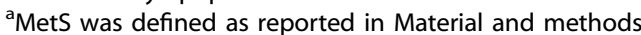

${ }^{\mathrm{b}} \mathrm{NAFLD}$ was defined as having hepatic fat content (HFF) $\geq 5 \%$ at $\mathrm{MRI}^{30-32}$

$\mathrm{HFF} \geq 5.0 \%$, our threshold criteria for the definition of NAFLD. Compared to girls, a significantly higher proportion of boys showed NAFLD $(P=0.028)$, even though this difference disappeared when adjusted for age, BMl, and pubertal stage $\left(P_{\text {adj }}=\right.$ $0.07)$. Boys showed significantly higher median ALT $(P=0.005)$ and $\gamma \mathrm{GT}(P=0.002)$ (Table 1$)$.

Metabolic and genetic predictors of HFF

Supplemental Table S1 (online) shows the results of univariate linear regression analysis associating HFF\% with demographic and biochemical variables. HFF\% was positively related with $\mathrm{BMI}$, glucose and insulin levels, MetS, ALT, AST, yGT, albumin and, negatively, with AST/ALT ratio.
To test whether genetic variants were associated with liver fat content, we first compared HFF\% between genotypes. The distribution of genotyped SNPs was in HWE (all $P>0.05$ ) and the minor allele frequency (MAF) for all variants was comparable to that reported in Europeans Non-Finnish. ${ }^{37}$ Overall, $122(53.1 \%)$ subjects were carriers of G PNPLA3 allele, $163(70.9 \%)$ of T MBOAT7 allele, $183(79.5 \%)$ of T GCKR allele, and 32 (13.9\%) of T TM6SF2 allele.

As reported in Fig. 1, carriers of $\mathrm{G}$ allele in PNPLA3, $\mathrm{T}$ allele in GCKR and T allele in TM6SF2 had higher HFF\% than wild-type carriers, even after adjustment for age, gender, BMI, pubertal stage, and $\mathrm{HOMA}_{\mathrm{IR}}\left(P_{\mathrm{adj}}<0.001, P_{\mathrm{adj}}<0.004\right.$, and $P_{\mathrm{adj}}=0.047$, respectively). We did not identify any association between HFF\% and MBOAT7 genotypes. Noteworthy, each copy of G PNPLA3 allele determined a significant twofold increase in HFF\% [median (interquartile range) $=6 \%(1.9-12.2)$ in CG vs. $10 \%(6.0-23.5)$ in GG $(P=0.019)]$.

Table 2 shows the contribution of genetic and non-genetic factors in predicting HFF. The model 2, which included age, gender, BMI, $\mathrm{HOMA}_{\mathrm{IR}}$, PNPLA3, GCKR, and TM6SF2 gene variants (Enter method), showed the highest ability in predict HFF\% explaining $24.8 \%$ of its variability $(P<0.001)$.

Notably, PNPLA3 variant appeared to be the single best predictor of HFF\% overcoming the potency of the other metabolic parameters. It is interesting to note that PNPLA3, GCKR, and TM6SF2 variants alone were able to explicate about $16.1 \%$ of HFF variation, a proportion higher than that due to metabolic factors alone $(8.7 \%)$. The results did not change even after including in the model MetS diagnosis as covariate.

\section{Predictors of NAFLD}

Supplemental Table S2 (online) shows the baseline characteristics and genotypes of children with and without the presence of hepatic steatosis. Children with NAFLD showed significantly higher prevalence of obesity $\left(P_{\text {adj }}=0.021\right)$ and MetS $(P=0.001)$ and about $54.3 \%$ of them had elevated ALT $\left(P_{\text {adj }} \leq 0.001\right)$. The rs738409 in PNPLA3 and rs58542926 in TM6SF2, but not GCKR and MBOAT7 gene variants, emerged as significantly associated with NAFLD $\left(P_{\text {adj }}<0.001\right.$ and $\left.P_{\text {adj }}=0.012\right)$. Notably, TM6SF2 167 EK + KK carriers had a threefold increased risk for hepatic steatosis (OR $3.1,95 \% \mathrm{Cl}: 1.3-7.1 ; P_{\text {adj }}=0.008$, data not shown) independently from age, gender, BMI, pubertal stage, and PNPLA3 I148M genotype. More importantly, homozygous PNPLA3 carriers (GG genotype) showed the highest risk of NAFLD (OR 14.9, 95\% Cl: 4.3-51.5; $P_{\text {adj }}<0.001$, data not shown).

When genetic factors were combined with demographic and metabolic variables (Table 3), the model including PNPLA3 and TM6SF2 variants and BMI had the highest discriminatory ability (all bootstrap $P_{\text {adj }} \leq 0.013$ ) in predicting the presence of NAFLD.

Comparing NAFLD children according to ALT levels, PNPLA3 rs738409 and BMI, but not TM6SF2 rs58542926 still emerged as predictors of NAFLD in those with elevated ALT (OR 6.0,95\% Cl: 3.0-11.9; $\left.P_{\text {adj }}<0.001\right)$. Conversely, TM6SF2 167 EK + KK genotype was the best predictor of NAFLD in the presence of normal ALT conferring fivefold increased risk of fatty liver $\left(P_{\mathrm{adj}}=0.001\right)$, higher than that due to PNPLA3 I148M or M148M genotypes (OR 2.1, 95\% Cl: 1.13-4.00; $\left.P_{\text {adj }}=0.018\right)$.

Development of a genetic score for NAFLD

Finally, we evaluated the cumulative effect of the four SNPs on the risk of NAFLD and we found that a weighted 4-SNPs GRS conferred the highest risk of hepatic steatosis (OR 63.6, 95\% Cl: 13.4-292.9; $\left.P_{\text {adj }}<0.001\right)$. In order to explore the robustness of this association, we conducted analysis by excluding one genetic variant at a time and we found that the 3-SNPs GRS without$M B O A T 7$ variant was able to explain the highest risk of NAFLD (OR 83.5, 95\% Cl: 16.9-411.5; $P_{\text {adj }}<0.001$, bootstrap $\left.P_{\text {adj }}=0.001\right)$. Moreover, the prevalence of NAFLD among children significantly 

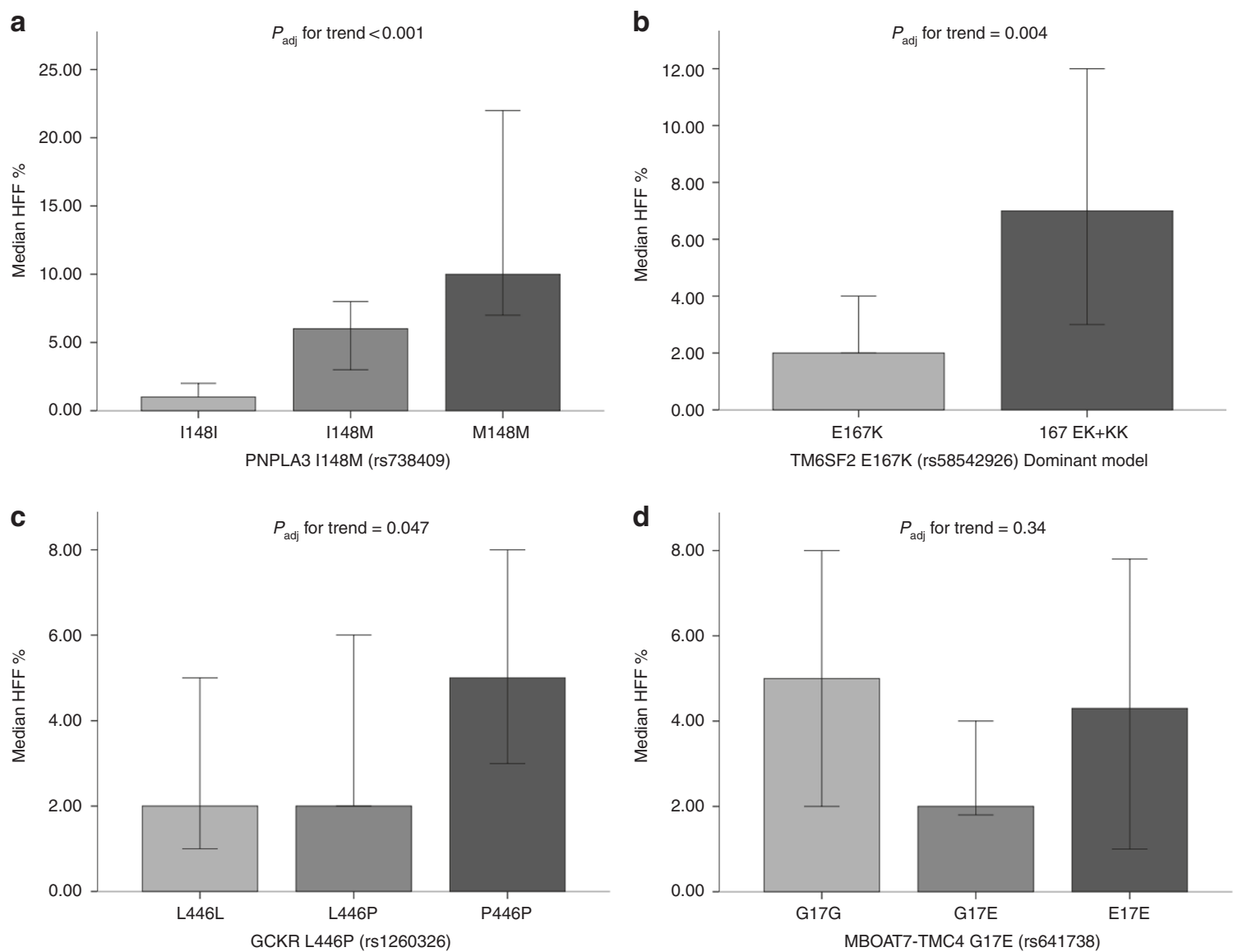

Fig. 1 Hepatic fat content according to genotypes. Linear regression analysis was used to evaluate associations between HFF\% and a PNPLA3 I148M, b TM6SF2 E167K, c GCKR L446P and d MBOAT7-TMC4 G17E genotypes. * $P_{\text {adj }}$ for trend shows the significance level of association tested by linear regression analysis adjusted for age, gender, pubertal stage, BMI, and $\mathrm{HOMA}_{\mathrm{IR}}$

Table 2. Multivariate models describing genetic and non-genetic predictors of hepatic fat content (HFF\%) in children

\begin{tabular}{|c|c|c|c|c|c|c|c|}
\hline & $R^{2}$ & $R^{2}$ variation & $F$-test & $\begin{array}{l}P \text {-value } \\
F \text {-test }\end{array}$ & $\beta$ & $95 \% \mathrm{Cl}$ & $P$-value \\
\hline Model 1 & 0.087 & 0.087 & 5.220 & $<0.001$ & & & \\
\hline Age & & & & & 0.072 & $-12.29-7.61$ & 0.64 \\
\hline Gender & & & & & -0.085 & $-4.47-0.94$ & 0.19 \\
\hline BMI & & & & & 0.113 & $-0.12-0.68$ & 0.17 \\
\hline $\mathrm{HOMA}_{\mathbb{R}}$ & & & & & 0.175 & $0.18-1.52$ & 0.01 \\
\hline Model 2 & 0.248 & 0.161 & 10.141 & $<0.001$ & & & \\
\hline Age & & & & & -0.029 & $-0.58-0.39$ & 0.69 \\
\hline Gender & & & & & -0.091 & $-4.38-0.57$ & 0.13 \\
\hline BMI & & & & & 0.179 & $0.07-0.81$ & 0.018 \\
\hline $\mathrm{HOMA}_{\mathrm{IR}}$ & & & & & 0.169 & $0.20-1.43$ & 0.009 \\
\hline $\begin{array}{l}\text { PNPLA3 variant } \\
\text { (additive model) }\end{array}$ & & & & & 0.347 & $3.60-7.36$ & $1 \times 10^{-7}$ \\
\hline $\begin{array}{l}\text { GCKR variant } \\
\text { (additive model) }\end{array}$ & & & & & 0.100 & $-0.30-3.31$ & 0.10 \\
\hline $\begin{array}{l}\text { TM6SF2 variant } \\
\text { (dominant model) }\end{array}$ & & & & & 0.155 & $1.10-8.08$ & 0.010 \\
\hline
\end{tabular}

increased along with increasing tertiles of 3-SNPs GRS (Fig. 2a) and the adjusted risk of hepatic steatosis raised up to 7.7 for GRS values $>0.3302$ (corresponding to the 3 th tertile) $\left(P_{\text {adj }}<0.001\right.$ Fig. 2b). The inclusion of MetS in the model did not change the power of weighted GRS in predicting fatty liver in children.

\section{DISCUSSION}

The present study was designed to weigh the impact of genetic and metabolic variables in liver fat accumulation in a cohort of children with obesity. Whereas increased body weight is a wellestablished determinant of fatty liver, the identification of factors interacting with obesity to produce a pro-steatogenic phenotype may have a clinical relevance.

Overall, we observed that genetic factors showed the strongest independent contribution to fatty liver and this effect was higher than that associated to insulin resistance, a typical obesity-linked metabolic abnormality. Indeed, we found that the variation of HFF was explained for $8.7 \%$ by metabolic factors and for $16.1 \%$ by the joint effect of PNPLA3, GCKR, and TM6SF2 variants. It is interesting to note that the rs $738409 \mathrm{G}$ allele in PNPLA3 showed the strongest effect with a standardized $\beta$-coefficient of 0.35 , larger than that conferred by the increasing of 1 standard deviation of $\mathrm{BMI}(\beta=$ $0.18)$ and insulin resistance $(\beta=0.17)$.

In support of this data, we also found that children carrying the PNPLA3 G and/or TM6SF2 T alleles have more than threefold higher risk of NAFLD than non-carriers. In contrast, the presence of hepatic steatosis was not significantly associated with age, gender, pubertal status or $\mathrm{HOMA}_{\mathrm{IR}}$, and only $\mathrm{BMI}$ and MetS emerged as independent metabolic predictor of NAFLD.

Obesity increases the risk of liver fat accumulation, ${ }^{4}$ but it is also evident that this measure alone is not sufficient for indicating increased HFF. Indeed, our data show that $87.9 \%$ of children with normal liver fat content were above the 95 th percentile of BMI. It is also important to note that, in a recent study carried out in a cohort of 2042 children followed up over 30 years, Suomela et al. ${ }^{22}$ 
demonstrated that a combined risk score based on childhood BMI, insulin levels, birth weight and the genetic variants in PNPLA3 and TM6SF2 was superior to a model including only BMI and insulin values in predicting adult fatty liver.

In the present study, we have considered genetic variants in PNPLA3, TM6SF2, GCKR, and MBOAT7 genes for their recognized role in determining NAFLD in adults. ${ }^{19}$ However, as illustrated in Supplemental Table S3 (online), few studies have comprehensively evaluated these susceptibility genes in children. In our cohort PNPLA3 and TM6SF2 variants were strongly associated with HFF\% as well as with NAFLD, while a much weaker association was detected with the common rs 1260326 GCKR variant. This is in contrast with our recent observations where the GCKR T allele was associated with significantly higher risk of NAFLD in adults. ${ }^{19}$ Even though this result could be due to the small sample size, data shown in Supplemental Table S3 (online) highlight that the association of GCKR variant with NAFLD in children is controversial. To interpret this discrepancy, we could speculate that the weak pro-steatogenic effect of GCKR in young people could depend on the shorter time of exposure to environmental factors interacting with the GCKR gene variant. In fact, it has been reported that the rs1260326 variant, which encodes for the GCKR P446L protein, deregulates glucose storage and disposal thus promoting de novo lipogenesis. ${ }^{38}$ Because carbohydrates are the upstream substrates in the glucokinase-regulated pathway, the effect of GCKR variant on the NAFLD susceptibility may be

Table 3. Genetic and non-genetic predictors of NAFLD in children

\begin{tabular}{lllll}
\hline Gene, SNP ID & $\beta$ & OR $(95 \% \mathrm{Cl})$ & $P$-value & $P_{\text {adj-value }}$ \\
\hline $\begin{array}{l}\text { PNPLA3, rs738409 } \\
\text { Additive model }\end{array}$ & 1.27 & $3.56(2.18-5.83)$ & $<0.001$ & 0.001 \\
TM6SF2, rs58542926 & 1.14 & $3.15(1.29-7.68)$ & 0.011 & 0.013 \\
$\begin{array}{l}\text { Dominant model } \\
\text { BMI }\left(\mathrm{kg} / \mathrm{m}^{2}\right)\end{array}$ & 0.16 & $1.17(1.08-1.27)$ & $<0.001$ & 0.011 \\
\hline
\end{tabular}

In the model were included: age (years), gender $(\mathrm{M} / \mathrm{F}), \mathrm{BMI}\left(\mathrm{kg} / \mathrm{m}^{2}\right)$, $\mathrm{HOMA}_{\mathrm{IR}}$, rs738409 PNPLA3, rs1230326 GCKR, rs641738 MBOAT7 (additive models), and rs58542926 TM6SF2 (dominant model) (forward Wald ratio method). Only significant variables were reported

$O R$ Odds ratio, $\mathrm{Cl}$ confidence interval

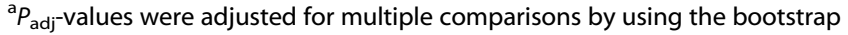
method mediated by the dietary intake of sugar. ${ }^{39}$ Therefore, a shorter exposure to sugar-enriched diet may account for the inconsistent results in the association between GCKR and fatty liver in childhood. Further investigations also considering the dietary carbohydrate intake are warranted to prove this hypothesis.

Moreover, we did not find any association between MBOAT7 gene variant and fatty liver accumulation. Three previous studies have explored this relation in obese children, but reporting inconsistent results. ${ }^{20,21,40}$ Two studies, where the effect of the rs641738 was explored in children evaluated by liver ultrasounds, did not find any independent association (see Supplementary Table S3, online). Conversely, Umano et al. ${ }^{40}$, which considered a different SNP in MBOAT7 gene, (rs626283) reported a positive association of this SNP with NMR-measured HFF\%, but only in the subgroup of Caucasian children. Even though rs626283 is in strong LD with rs641738, it has been consistently associated with more advanced liver disease. ${ }^{41}$ It must be noted that compared to ours, children enrolled in the Umano's study showed markedly higher indices of obesity (BMI $32.9 \pm 7.5 \mathrm{~kg} / \mathrm{m}^{2}$ ) and insulin resistance, thus leaving the possibility that they were presenting a more advanced liver damage. To this regard, the slightly higher levels of ALT reported in their homozygous carriers for rs626283 (ALT $30.2 \pm 26.5 \mathrm{U} / \mathrm{L}$ ) as compared to our homozygotes (ALT $23.0 \pm 13.1 \mathrm{U} / \mathrm{L}$ ) is highly suggestive of this possibility.

In line with previous studies in adults, we found that variants in the susceptibility genes act in an additive fashion in promoting liver steatosis in children with obesity. By using a weighted GRS, we showed that individuals carrying PNPLA3, GCKR, and TM6SF2 gene variants have about eightfold higher risk to develop fatty liver than non-carriers. There are few attempts in the literature to develop a GRS to illustrate the combined effect of genetic variants on liver fat accumulation in children. Walker et al. ${ }^{23}$ reported that a GRS comprising PNPLA3 and APOC3, but not the other susceptibility alleles, accounted for $12 \%$ of the variance in HFF and children with four risk alleles had threefold higher liver fat than non-variant carriers. The higher predictive power of our GRS is not easy to explain. The fact that Walker et al. included Hispanic children, which are very vulnerable to obesogenic environmental factors, might have limited the impact of genetic factors on the hepatic fat content variation. In fact, in the Walker's cohort BMI and ALT showed the highest predictive power of HFF. ${ }^{23}$ Our findings indicate that a comprehensive weighted GRS significantly improves the risk classification of increased fat content also in children with obesity. Further confirmation in larger cohort of a

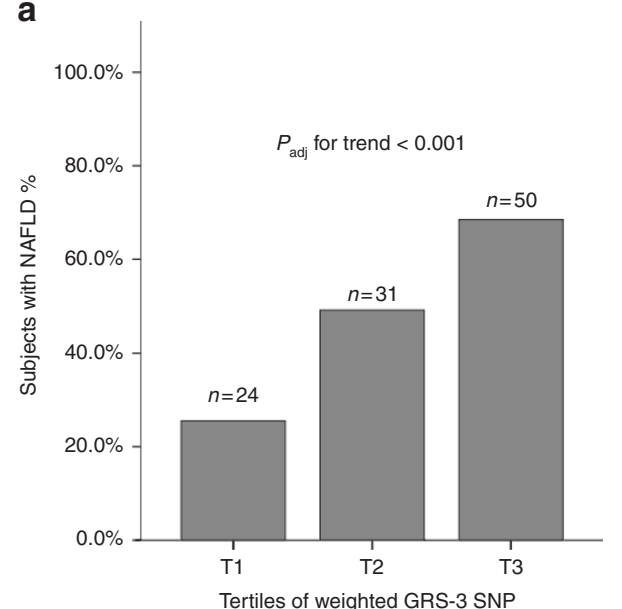

b

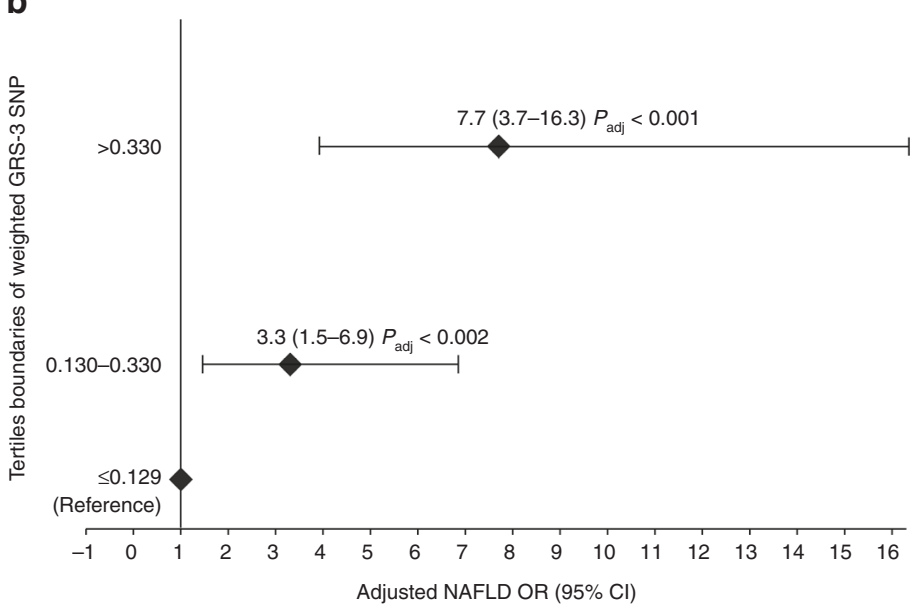

Fig. 2 Association of weighted GRS 3-SNP with the risk of NAFLD. a Distribution of tertiles of weighted 3-SNP GRS in NAFLD patients; $\mathbf{b}$ NAFLD ORs adjusted for age, gender, BMI, and HOMA $A_{I R}$ across tertiles of weighted 3-SNP GRS. $P_{\text {adj }}$ for trend was adjusted for age, gender, BMI, $\mathrm{HOMA}_{\mathrm{R}}$, and tertiles of weighted 3-SNPs GRS ( $X^{2}$ Pearson followed by Stepwise regression analysis); NAFLD ORs were adjusted for age, gender, $B M I, H O M A_{I R}$, and tertiles of weighted GRS (regression analysis, enter method) 
children with different ethnicity is needed. More importantly, since these genes have been also reported as modifiers of liver steatosis progression, ${ }^{42}$ their evaluation in combination with liver histology, measures of fibrogenesis or inflammatory biomarkers, is needed to better understand which genes play roles in the initial steps of steatogenesis as well as in the fibro-inflammatory evolution of NAFLD in children.

The present study has several strengths such as the careful metabolic evaluation of participants and the use of MRI to obtain the best accurate quantitative measurement of liver fat content. It has also limitations to be acknowledged. The cohort size is small, and we do not have longitudinal data, which might help in assessing the long-term effect of metabolic and genetic factors. Moreover, the lack of biochemical markers of fibrosis, as well as of measurement of visceral obesity or evaluation of other obesity-related factors [i.e., fructose-exceedingly rich diets or fructose intolerance ${ }^{3,43}$ ] did not permit a careful weighing of additional factors implicated in NAFLD or liver damage development.

In addition, we were not able to assess NAFLD by liver biopsy, but previous published studies have reported a high correlation between MRI-derived HFF\% and histology in children. ${ }^{31,32}$ Moreover, in developing the weighted GRS, we have used $\beta$-coefficients obtained in adults ${ }^{19,35}$ which not necessarily may be applicable to a children population. Finally, an additional limitation of our study was the lack of replication in an independent sample. However, it must to be noted that we have considered well known genetic determinants of NAFLD not requiring replication.

In conclusion, we have shown that genetic factors have the greatest impact in determining the amount of liver fat content in children, higher than that attributable to metabolic alterations associated with obesity. Due to the large diffusion of obesityrelated liver disease at all ages, there is a strong need to identify genotypes that correlate especially to pediatric NAFLD/NASH phenotype in order to decrease future fatty liver-related morbidity and develop a comprehensive risk factor panels finalized to the prediction of NAFLD risk at individual level. If definitely confirmed, this approach would provide a great help in designing early preventive intervention in high-risk children with obesity to prevent adult complication of NAFLD.

\section{ACKNOWLEDGEMENTS}

This study was supported by grant from Sapienza University of Rome (2016).

\section{AUTHOR CONTRIBUTIONS}

A.D.C. performed genetic analysis, analyzed the data, generated figures, performed literature search, and prepared the manuscript; L.P. designed the study, collected the sample cohort, interpreted the data, and revised the manuscript; C.C. designed the study, interpreted the data, and revised the manuscript; F.M.P. collected the sample cohort and revised the manuscript; F.C. carried out the laboratory measurements and revised the manuscript; A.A. carried out the laboratory measurements and revised the manuscript; L.D.E. analyzed the data, prepared the manuscript, and performed literature search; M.D.M. carried out the MRI evaluation and revised the manuscript; MA designed the study, interpreted the data and prepared the manuscript. All authors edited and approved the final manuscript as written and take responsibility for its content.

\section{ADDITIONAL INFORMATION}

The online version of this article (https://doi.org/10.1038/s41390-019-0303-1) contains supplementary material, which is available to authorized users.

Competing interests: The authors declare no competing interests.

Publisher's note: Springer Nature remains neutral with regard to jurisdictional claims in published maps and institutional affiliations.

\section{REFERENCES}

1. Mencin, A. A. \& Lavine, J. E. Advances in pediatric nonalcoholic fatty liver disease. Pediatr. Clin. North Am. 58, 1375-1379 (2011).

2. Bugianesi, E. et al. Low birthweight increases the likelihood of severe steatosis in pediatric non-alcoholic fatty liver disease. Am. J. Gastroenterol. 112, 1277-1286 (2017).

3. Clemente, M. G., Mandato, C., Poeta, M. \& Vajro, P. Pediatric non-alcoholic fatty liver disease: Recent solutions, unresolved issues, and future research directions. World J. Gastroenterol. 22, 8078-8093 (2016).

4. Anderson, E. L. et al. The prevalence of non-alcoholic fatty liver disease in children and adolescents: a systematic review and meta-analysis. PLOS ONE 10, e0140908 (2015).

5. Mencin, A. A. \& Lavine, J. E. Non-alcoholic fatty liver disease in children. Curr. Opin. Clin. Nutr. Metab. Care. 14, 151-157 (2011).

6. Chalasani, N. et al. The diagnosis and management of non-alcoholic fatty liver disease: practice guideline by the American Association for the Study of Liver Diseases, American College of Gastroenterology and the American Gastroenterological Association. Hepatology 55, 2005-2023 (2012).

7. Benedict, M. \& Zhang, X. Non-alcoholic fatty liver disease: an expanded review. World J. Hepatol. 9, 715-732 (2017).

8. Marchesini, G. et al. Non-alcoholic fatty liver disease: a feature of the metabolic syndrome. Diabetes 50, 1844-1850 (2001).

9. Gruben, N., Shiri-Sverdlov, R., Koonen, D. P. \& Hofker, M. H. Nonalcoholic fatty liver disease: A main driver of insulin resistance or a dangerous liaison? Biochim Biophys. Acta 1842, 2329-2343 (2014).

10. Nobili, V. et al. A 360-degree overview of paediatric NAFLD: recent insights. J. Hepatol. 58, 1218-1229 (2013).

11. Dongiovanni, P. \& Valenti, L. Genetics of nonalcoholic fatty liver disease. Metabolism 65, 1026-1037 (2016).

12. Romeo, S. et al. Genetic variation in PNPLA3 confers susceptibility to nonalcoholic fatty liver disease. Nat. Genet. 40, 1461-1465 (2008).

13. Sookoian, S. \& Pirola, C. J. Meta-analysis of the influence of $1148 \mathrm{M}$ variant of patatin-like phospholipase domain containing 3 gene (PNPLA3) on the susceptibility and histological severity of nonalcoholic fatty liver disease. Hepatology 53, 1883-1894 (2011).

14. Pirazzi, C. et al. Patatin-like phospholipase domain-containing 3 (PNPLA3) I148M (rs738409) affects hepatic VLDL secretion in humans and in vitro. J. Hepatol. 57, 1276-1282 (2012).

15. Kozlitina, J. et al. Exome-wide association study identifies a TM6SF2 variant that confers susceptibility to nonalcoholic fatty liver disease. Nat. Genet. 46, 352-356 (2014).

16. Mahdessian, $\mathrm{H}$. et al. TM6SF2 is a regulator of liver fat metabolism influencing triglyceride secretion and hepatic lipid droplet content. Proc. Natl Acad. Sci. USA 111, 8913-8918 (2014).

17. Speliotes, E. K. et al. Genome-wide association analysis identifies variants associated with non- alcoholic fatty liver disease that have distinct effects on metabolic traits. PLoS Genet. 7, e1001324 (2011).

18. Mancina, R. M. et al. The MBOAT7-TMC4 variant rs641738 increases risk of nonalcoholic fatty liver disease in individuals of European descent. Gastroenterology 150, 1219-1230 (2016).

19. Di Costanzo, A. et al. Evaluation of polygenic determinants of non-alcoholic fatty liver disease (NAFLD) by a candidate genes resequencing strategy. Sci. Rep. 27, 3702 (2018).

20. Lin, Y. C., Chang, P. F., Chang, M. H. \& Ni, Y. H. Genetic determinants of hepatic steatosis and serum cytokeratin-18 fragment levels in Taiwanese children. Liver Int. 38, 1300-1307 (2018).

21. Di Sessa, A. et al. The membrane-bound O-Acyltransferase7 rs641738 variant in pediatric nonalcoholic fatty liver disease. J. Pediatr. Gastroenterol. Nutr. 67, 69-74 (2018).

22. Suomela, E. et al. Childhood predictors of adult fatty liver. The cardiovascular risk in Young Finns Study. J. Hepatol. 65, 784-790 (2016).

23. Walker, R. W. et al. Genetic and clinical markers of elevated liver fat content in overweight and obese Hispanic children. Obesity 21, E790-E797 (2013).

24. Cole, T. J., Bellizzi, M. C., Flegal, K. M. \& Dietz, W. H. Establishing a standard definition for child overweight and obesity worldwide: international survey. BMJ 320, 1240-1243 (2000).

25. Pacifico, L. et al. Functional and morphological vascular changes in pediatric nonalcoholic fatty liver disease. Hepatology 52, 1643-1651 (2010).

26. Marshall, W. A. \& Tanner, J. M. Variations in the pattern of pubertal changes in boys. Arch. Dis. Child 45, 13-23 (1970).

27. Pacifico, L. et al. Association of serum triglyceride-to-HDL cholesterol ratio with carotid artery intima-media thickness, insulin resistance and nonalcoholic fatty liver disease in children and adolescents. Nutr. Metab. Cardiovasc Dis. 24, 737-743 (2014).

28. Cook, S., Auinger, P. \& Huang, T. T. Growth curves for cardio-metabolic risk factors in children and adolescents. J. Pediatr. 155, S6.e15-26 (2009). 
29. National High Blood Pressure Education Program Working Group on High Blood Pressure in Children and Adolescents. The fourth report on the diagnosis, evaluation, and treatment of high blood pressure in children and adolescents. Pediatrics 114(Suppl.2), 555-576 (2004).

30. Pacifico, L. et al. The impact of nonalcoholic fatty liver disease on renal function in children with overweight/obesity. Int J. Mol. Sci. 17, 1218 (2016).

31. Pacifico, L. et al. T1-weighted dual-echo MRI for fat quantification in pediatric nonalcoholic fatty liver disease. World J. Gastroenterol. 17, 3012-3019 (2011).

32. Di Martino, M. et al. Comparison of magnetic resonance spectroscopy, proton density fat fraction and histological analysis in the quantification of liver steatosis in children and adolescents. World J. Gastroenterol. 22, 8812-8819 (2016).

33. Schwimmer, J. B. et al. SAFETY study: alanine aminotransferase cutoff values are set too high for reliable detection of pediatric chronic liver disease. Gastroenterology 138, 1357-1364 (2010).

34. Di Costanzo, A. et al. Non-alcoholic fatty liver disease and subclinical atherosclerosis: a comparison of metabolically-versus genetically-driven excess fat hepatic storage. Atherosclerosis 257, 232-239 (2017).

35. Dongiovanni, P. et al. Causal relationship of hepatic fat with liver damage and insulin resistance in nonalcoholic fatty liver. J. Intern Med. 283, 356-370 (2018).

36. Rijks, J. et al. Glycaemic profiles of children with overweight and obesity in free-living conditions in association with cardiometabolic risk. Sci. Rep. 6, 31892 (2016).

37. Karczewski, K. J. et al. The ExAC browser: displaying reference data information from over 60000 exomes. Nucleic Acids Res. 45(Database issue), D840-D845 (2017).

38. Rees, M. G. et al. Cellular characterisation of the GCKR P446L variant associated with type 2 diabetes risk. Diabetologia 55, 114-122 (2012).

39. Lin, Y. C., Chang, P. F., Chang, M. H. \& Ni, Y. H. Genetic variants in GCKR and PNPLA3 confer susceptibility to non-alcoholic fatty liver disease in obese individuals. Am. J. Clin. Nutr. 99, 869-874 (2014)
40. Umano, G. R. et al. The rs626283 variant in the MBOAT7 gene is associated with insulin resistance and fatty liver in caucasian obese youth. Am. J. Gastroenterol. 113, 376-383 (2018).

41. Buch, S. et al. A genome-wide association study confirms PNPLA3 and identifies TM6SF2 and MBOAT7 as risk loci for alcoholrelated cirrhosis. Nat. Genet. 47, 1443-1448 (2015).

42. Scott, E. \& Anstee, Q. M. Genetics of alcoholic liver disease and non-alcoholic steatohepatitis. Clin. Med. 18(Suppl 2), s54-s59 (2018).

43. D'Aniello, R. et al. Emerging pathomechanisms involved in obesity. J. Pediatr. Gastroenterol. Nutr. 60, 113-119 (2015).

(i) $९$ Open Access This article is licensed under a Creative Commons Attribution-NonCommercial-NoDerivatives 4.0 International License which permits any non-commercial use, sharing, distribution and reproduction in any medium or format, as long as you give appropriate credit to the original author(s) and the source, and provide a link to the Creative Commons license. You do not have permission under this license to share adapted material derived from this article or parts of it. The images or other third party material in this article are included in the article's Creative Commons license, unless indicated otherwise in a credit line to the material. If material is not included in the article's Creative Commons license and your intended use is not permitted by statutory regulation or exceeds the permitted use, you will need to obtain permission directly from the copyright holder. To view a copy of this license, visit http://creativecommons.org/licenses/by-nc-nd/4.0/.

(c) The Author(s) 2019 